Check for updates

Cite this: Nanoscale Adv., 2019, 1, 2258

\title{
Unraveling the photoactive annihilation mechanism of nanostructures as effective green tools for inhibiting the proliferation of the phytopathogenic bacterium Pseudomonas syringae
}

\author{
Sergio Casas-Flores, (D) a Ruth B. Domínguez-Espíndola, (D) ${ }^{b}$ Roberto Camposeco- \\ solis, ${ }^{a}$ Olga A. Patrón-Soberano (D) ${ }^{a}$ and Vicente Rodríguez-González (D) *a
}

\begin{abstract}
The infectious proliferation of phytopathogenic microorganisms depends on a complex sequence of biological events involving host defense, environmental conditions, and chemical and physical interactions between the surface of a plant and microorganisms, which in numerous cases display resistance to conventional microbicides. Among these microorganisms, Pseudomonas syringae ( $P$. syringae) is a Gram-negative bacterium that attacks wounded parts of plants before invading healthy tissues. In order to control $P$. syringae, considering it to be a phytopathogenic model, an effective method featuring silver nanoparticles (AgNPs) functionalized on titanate nanotubes (Nts) used as photoactive antibacterial agents was investigated to understand the effective photoactive annihilation mechanism. The high dispersion of AgNPs over the Nts boosted charge carrier separation by generating reactive oxygen species (ROS) under visible-light, which stressed the bacteria and enhanced the biocidal effect by quickly preventing the rod-shaped $P$. syringae bacteria from proliferating. Biological transmission and scanning electron microscopy revealed damaged $P$. syringae cells that underwent the formation of outer membrane vesicles, caused by photo-assisted annihilation, which is considered to be an indication of a critical defense mechanism. The unusual synergistic properties of the Nts, and their low cost and practical synthesis, made these nanocomposites promising green tools that can positively and swiftly photokill $P$. syringae within $30 \mathrm{~min}$.
\end{abstract}

Received 26th October 2018
Accepted 14th April 2019
DOI: 10.1039/c8na00307f
rsc.li/nanoscale-advances

\section{Introduction}

Nowadays, the demand for grains, fruits, vegetables and other important crops has expanded because of the enormous growth in human population. Huge expanses of land are dedicated to planting crops in order to meet the high demand for grains, vegetables, and fruits for human consumption and livestock feeding; in addition, some plants are cultivated all through the year in large greenhouses. To accomplish these goals, chemicals such as plant growth regulators, fertilizers, and pesticides to control the production of these plant-based foods are required. ${ }^{1,2}$ Mass cultivation is susceptible to phytopathogenic microorganisms that cause many different plant diseases which can devastate agricultural crops. In this sense, necrosis, tissue maceration,

${ }^{a} I P I C Y T$, Instituto Potosino de Investigación Científica y Tecnológica, Division de Biologia Molecular, División de Materiales Avanzados, Camino a la Presa San José 2055, Lomas 4a. sección 78216, San Luis Potosí, S.L.P., México. E-mail: vicente. $r d z @ i p i c y t . e d u . m x$

${ }^{b}$ Posgrado en Ingeniería y Ciencias Aplicadas, Universidad Autónoma del Estado de Morelos, Cuernavaca, Morelos C.P. 62209, Mexico wilting, and hyperplasia are just some of these diseases. Fungi and bacteria reproduce more easily in plants close to each other. Many of the phytopathogenic Pseudomonas spp. first induce water-soaked injuries in the host plant followed by necrosis and chlorosis of the tissues. ${ }^{2-4}$ More specifically, Pseudomonas syringae is a hemibiotrophic bacterial pathogen that affects a wide variety of plants, causing blight, speck, and spot diseases in many important agricultural crops including tomato, soybean, rice, and tobacco, among others. ${ }^{2}$ P. syringae can be differentiated into more than 50 pathovars according to the disease caused by the strain in plants. ${ }^{5}$ The multiple strains of this species cause diseases in more than 180 plant species. ${ }^{6} P$. syringae is divided into 8 genomospecies and 13 phylogroups. ${ }^{7}$ The interactions between plants and $P$. syringae have been extensively studied, but the defense mechanisms of plant during inactivation have been scarcely investigated. ${ }^{3,8,9}$ Diverse alternatives have been considered for inhibiting the proliferation of plant pathogenic microorganisms. The indiscriminate use of chemicals and pesticides represents a risk for human and animal consumers as well as increasing the final cost of the vegetables, grains, and 
fruits. ${ }^{\mathbf{1 , 2 , 9 - 1 1}}$ Detrimental effects such as the acceleration of soil erosion, pollution of water bodies like rivers, lagoons, etc., and air pollution have to be considered. Other methods to control infections are the use of oxidizing disinfectants such as ozone and chlorine dioxide, UV light treatment, chlorination and peroxide oxidation. ${ }^{2,12-15}$ Most chemicals, bactericides and fungicides react with organic matter and other precursors to form regulated and emerging disinfection by-products, which can be associated with cancer or other human pathologies. ${ }^{\mathbf{1 4 , 1 5}}$ The most recent method to control phytopathogenic bacteria is the use of transgenic products like cotton, tomato, soybean, potato, etc. Transgenic plants have caused controversy because people are uncertain about their safety and regulatory issues, and changes in their nutritional properties and taste, ${ }^{\mathbf{1 6}}$ as well as in regulatory issues.

On the other hand, nanomaterials have emerged as a promising alternative to control the infection of plants by several microorganisms. ${ }^{17,18}$ They present a non-invasive way to battle infection, with practically no generation of toxic byproducts, and an eco-friendly way to control pests that could become of relevance in the near future. ${ }^{19}$ Several nanomaterials have been tested for the annihilation of pathogenic and phytopathogenic microorganisms, some with biocidal properties and others which are catalytically active. ${ }^{17-19}$ In this sense, titanium dioxide with silver nanoparticles is the most studied photoactive composite, which increases the biocidal efficiency against pathogenic bacteria such as Staphylococcus aureus, Salmonella spp., and Escherichia coli. Research studies have been focused on the evaluation of survival in terms of colony-forming units (CFUs) as a function of time..$^{\mathbf{2 0 2 1}}$ It has also been reported that reactive oxygen species (ROS) are responsible for photokilling mediated by UV active materials together with the biocidal activity of silver nanoparticles. Only a few researchers have studied the microorganism-nanomaterial interactions, morphology of microorganisms and materials, and wavelength of the lamps to get more insights into the way in which photo-assisted annihilation works..$^{22-25}$

In the present study, the sustainable synthesis of silver protonated nanotubes was evaluated for the annihilation of the plant pathogen $P$. syringae under visible light irradiation. The surface activity and structural properties of the silver nanocomposites were characterized by scanning electron microscopy (SEM), transmission electron microscopy (TEM), high angular annular dark-field scanning transmission electron microscopy (HAADF-STEM), X-ray diffraction (XRD), and ultraviolet-visible diffuse reflectance spectroscopy (UV-vis DRS), and directly correlated with the bacterial morphology. This study presents a concise discussion on assessing the effective inhibition of the proliferation and photokilling of the phytopathogenic bacteria by means of a reproducible biological protocol for sample preparation for electron microscopy techniques (SEM and TEM) that allow the monitoring of morphology changes of the microorganisms during photocatalytic inactivation with different AgNP loadings on the surface of photoactive nanostructures.

\section{Experimental}

\subsection{Synthesis of titanate nanotubes}

The procedure involved the addition of $2 \mathrm{~g}$ of commercial $\mathrm{TiO}_{2}$ known as P25 (Degussa, Rutile : Anatase, 85 : 15, 99.9\%, $\sim 20 \mathrm{~nm}$ crystallite size, CAS: $13463-67-7$ ) into $50 \mathrm{~mL}$ of a $10 \mathrm{M}$ alkaline solution of $\mathrm{NaOH} .^{22}$ The slurry was placed in an ultrasonic bath for 5 min to eliminate aggregates and then stirred at room temperature for $10 \mathrm{~min}$. The mixture was placed carefully in a $150 \mathrm{~mL}$ Teflon vessel and heated in a microwave reactor (MWO-1000S, EYELA, Japan). The temperature was fixed at $150{ }^{\circ} \mathrm{C}$ for $5 \mathrm{~h}$, at $400 \mathrm{rpm}$, and a maximum adjustable microwave irradiation power of $195 \mathrm{~W}$ was used. After that, the precipitate was cooled to room temperature and neutralized with a $5 \mathrm{~N}$ HCl solution until $\mathrm{pH} 7$ was reached. Then, the precipitated powders were filtered and dried overnight at $90{ }^{\circ} \mathrm{C}$. The as-obtained nanotubes were identified as Nts.

\subsection{Photochemical deposition of silver nanoparticles on the Nt surface}

The photodeposition of AgNPs was carried out as follows: $100 \mathrm{~mL}$ of an ethanol solution of $\mathrm{AgNO}_{3}$ was prepared by using three silver loadings $(0.5,1$, and $3 \mathrm{wt} \%)$. Then, a suitable amount of titanates was gently added to the solution. The slurry was maintained for $10 \mathrm{~min}$ under magnetic stirring and immediately placed for $10 \mathrm{~min}$ in an ultrasonic bath at room temperature. The slurry was kept under magnetic stirring for $1 \mathrm{~h}$ under dark conditions to facilitate the adsorption of silver cations; afterwards, the slurry was irradiated with UV-C light for $2 \mathrm{~h}$ to achieve the reduction of silver cations into highly dispersed AgNPs. The resulting powders were filtered and dried overnight at $90{ }^{\circ} \mathrm{C}$. The obtained powders were identified as $\mathrm{Nt} x \mathrm{Ag}$, where $x$ represents the silver loading in wt\%.

\subsection{Photoassisted annihilation of $P$. syringae cells}

The photocatalytic annihilation of $P$. syringae $p v$ tomato DC3000 (Pto DC3000) was achieved as follows: all Pyrex glass materials and apparatus used in these experiments were sterilized at $121{ }^{\circ} \mathrm{C}$ for $45 \mathrm{~min}$ using an autoclave. $P$. syringae was routinely grown on King B Agar medium at $28{ }^{\circ} \mathrm{C}$ for $24 \mathrm{~h}$. Afterwards, bacterial cells were collected with sterilized saline water and counted in a hemocytometer. All photocatalytic inactivation experiments were carried out in $30 \mathrm{~mL}$ Petri dishes $(90 \times 15 \mathrm{~mm})$ with constant magnetic stirring and irradiation with a halogen lamp (Eco cool halogen shine 30W, Yazawa Corp., Japan) to provide simulated solar light irradiation in the presence of $30 \mathrm{mg}$ of silver-nanotube composites. At fixed time intervals, samples were taken from the suspension, diluted and plated on King B plates in triplicate. After $24 \mathrm{~h}$ of incubation at $28{ }^{\circ} \mathrm{C}$ in the dark, the number of viable cells was determined as colony-forming units (CFUs) that correspond to at least one surviving cell. The number of colonies in a sample must be approximately 280 to ensure enumeration and reproducibility. 


\subsection{Photo-oxidation of Rhodamine B under visible light}

The 0D silver-nanotube composites were evaluated in the photocatalytic decomposition of Rhodamine B dye (RhB) using a homemade Pyrex reactor at room temperature, which was irradiated with a pen-ray visible lamp (450 nm) under dark conditions. For the photocatalytic tests, $50 \mathrm{mg}$ of catalyst were used. The aqueous solution contained $20 \mathrm{ppm}$ of RhB. Before the visible lamp was turned on, the system was placed in the dark for $1 \mathrm{~h}$ to achieve adsorption-desorption equilibrium. The photoactivity degradation rate was recorded by measuring the intensity of the main absorption band of RhB as a function of the irradiation time. Atmospheric air was bubbled during the photocatalytic experiments $\left(10 \mathrm{~mL} \min ^{-1}\right)$. At given time intervals, samples were extracted and then monitored with an UV-vis spectrophotometer $(5000$ UV-vis-NIR, Agilent Technologies).

\subsection{Characterization of silver-nanotube composites ( $\mathrm{Nt} x \mathrm{Ag}$ )}

The silver-nanotube composites ( $\mathrm{Nt} x \mathrm{Ag}$ ) were characterized by HRTEM. HRTEM images were obtained with a FEI Tecnai F30 microscope equipped with a tungsten field emission gun operated at $300 \mathrm{keV}$. HAADF imaging was performed in STEM mode. The semi-quantitative composition of the samples was determined by EDS with an EDAX spectrometer coupled to a TEM. The powdered samples were ultrasonically dispersed in isopropyl alcohol and supported on holey carbon-coated copper grids. UV-vis DRS spectra and band gap energy data were obtained using a UV-vis-NIR Agilent Technologies model Cary 5000 Series spectrophotometer equipped with an integrating sphere. X-ray diffraction patterns were obtained using a SmartLab RIGAKU X-ray diffractometer with $\mathrm{Cu} \mathrm{K} \alpha$ radiation $(1.5404 \AA$ and $1.5406 \AA$ ), operated at $35 \mathrm{kV}$ and $25 \mathrm{~mA}$.

\subsection{P. syringae characterization}

Cell samples were collected in a pellet at representative time intervals during and before photocatalytic inactivation. The bacterial pellets were fixed in $3 \%$ glutaraldehyde in a $100 \mathrm{mM}$ sodium phosphate buffer (PBS) at $\mathrm{pH} 7.4$ and $4{ }^{\circ} \mathrm{C}$ for $24 \mathrm{~h}$; the samples were carefully washed three times with PBS for $15 \mathrm{~min}$ each time. Afterwards, the samples were gradually dehydrated by using ethanol:water $(30: 70, \mathrm{v} / \mathrm{v})$ to absolute ethanol for 10 min with each concentration, and washed twice with absolute ethanol, each washing lasting $15 \mathrm{~min}$. The critical point drying process was performed cautiously in a Tousimis SamdriPVT-3D, and the dry samples were mounted to perform gold sputter coating using Cressington apparatus Model 108auto and examined using an FEI model Quanta 200 Scanning Electron Microscope. The electron microscope was adjusted to 25 $\mathrm{kV}$, spot 5.3, and WD $10 \mathrm{~mm}$, taking micrographs with an Everhart Thornley Detector (ETD).

The ultrastructure of $P$. syringae was observed with negative staining by JEOL 200CX TEM. Bacteria treated with silvernanotube composites at different time intervals were $2 \%$ uranyl acetate contrasted.

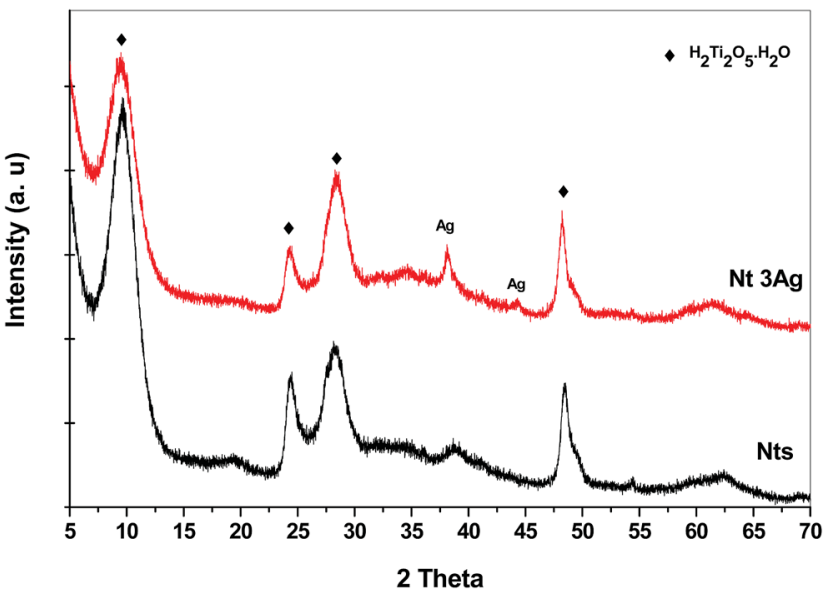

Fig. 1 XRD patterns for selected silver-nanotube structures.

\section{Results and discussion}

The XRD patterns for the different AgNP loadings on Nts are shown in Fig. 1. The XRD patterns show the characteristic lowcrystallinity for titanate materials, ${ }^{26}$ in which all reflections can be attributed to the orthorhombic phase of titanate nanotubes, $\mathrm{H}_{2} \mathrm{Ti}_{2} \mathrm{O}_{5} \cdot \mathrm{H}_{2} \mathrm{O}$ (JCPDS 47-0124), and the crystalline phase of metallic AgNPs (JCPDS 04-0783) (Fig. 1). Hydrogen titanate is the main crystalline phase in the nanocomposites. At a low content of silver loading, no reflection peak characteristic of metallic silver was detected, presumably due to the nanosize of the highly dispersed AgNPs. For $3 \mathrm{wt} \%$ of AgNP loading, two slight peaks ascribed to metallic silver were identified. This could also indicate that no agglomeration of AgNPs in clusters occurred during the photodeposition method of AgNPs, allowing high monodispersion on the nanotube surface.

The morphologies of the titanate nanotubes were confirmed through TEM and HRTEM (Fig. 2a and b). The TEM images obtained for the one-dimensional Nt and Nt3Ag materials are shown in Fig. $2 \mathrm{a}$ and $\mathrm{b}$, in which the shape of the nanotubes is clearly observed. The Nt diameter was 10-14 nm, with a length of $\sim 400$ to $600 \mathrm{~nm}$. On the other hand, it was difficult to distinguish some silver nanoparticles functionalized on the surfaces of the titanate nanotubes due to their nanosize; for this reason, it was necessary to use HAADF-STEM observations. In the literature, hydrothermally synthesized titanate nanotubes have exhibited diameters of about $15 \mathrm{~nm}$ and lengths from 10 to $100 \mathrm{~nm} .^{23-26}$

HRTEM observations of Nts in Fig. $2 b$ indicate that the nanotubes are generally three- to four-layered with an interlayer separation of $\sim 0.72 \mathrm{~nm}$, and that the titanate nanotubes have almost uniform inner diameters of $\sim 4 \mathrm{~nm}$ and outer diameters of $\sim 12 \mathrm{~nm}$. The formation mechanism of nanotubes from commercial $\mathrm{TiO}_{2}$ anatase in an alkaline ambient medium has been described elsewhere. ${ }^{26}$ On the other hand, it has been stated that the recrystallization of anatase and rutile phases is possible, in a complete transformation, due to which very small peaks attributed to the rutile phase have appeared in some cases in the XRD patterns. In our work, HAADF-STEM 

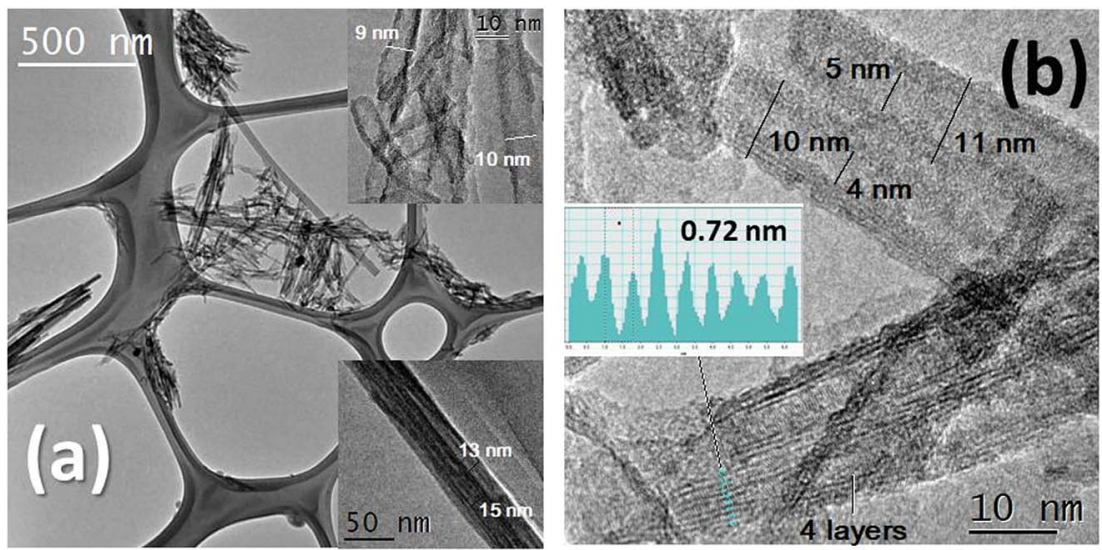

Fig. 2 TEM images of (a) synthesized silver nanotube composites, bundles of NtAg, with the inset showing the zoomed-in view of the nanotube morphology and (b) the HRTEM image of Nt3Ag showing inner and outer diameters and the interlayer distance.
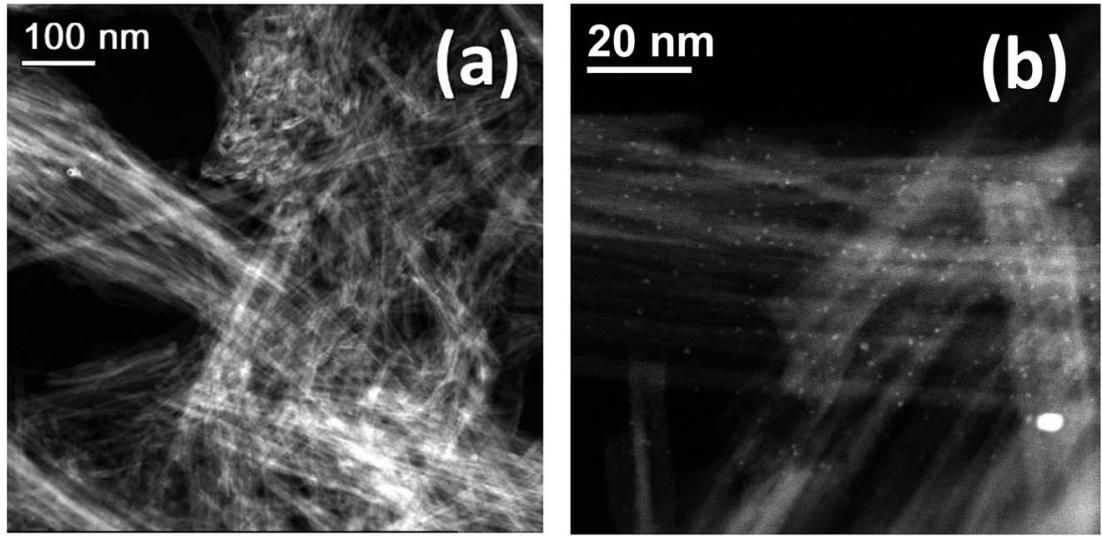

Fig. 3 HAADF-STEM images of (a) translucent bundles of the silver nanotube composite, Nt3Ag, and (b) the HAADF-STEM image of Nt3Ag showing highly-monodispersed AgNPs on the nanotube surface.

helped to identify more clearly the surface AgNPs on the titanate nanotubes, confirming their high monodispersion on the surface, as suggested by XRD patterns. Fig. 3a shows translucent bundles of the silver-nanotube composite, in which with more magnification, the AgNPs are observable. Fig. 3b shows that some silver nanoparticles were photodeposited specifically on the titanate surface with an average nanoparticle size of $4 \mathrm{~nm}$.

\subsection{Photoassisted annihilation of $P$. syringae}

The photoassisted annihilation of $P$. syringae bacteria under visible light irradiation by the photocatalysts as a function of time is shown in Fig. 4a for the three as-prepared silvertitanates, titanates alone and the commercial $\mathrm{TiO}_{2}-\mathrm{P} 25$. $\mathrm{Nt} 1 \mathrm{Ag}$ shows complete annihilation within $20 \mathrm{~min}$, followed by $\mathrm{Nt3Ag}$, which accomplished complete annihilation within 45 min. Commercial $\mathrm{TiO}_{2}$-P25 showed low photoactivity, only achieving $5 \%$ annihilation after $60 \mathrm{~min}$. The control growth test is also displayed. As shown in Fig. $4 \mathrm{~b}, \mathrm{Nt} 1 \mathrm{Ag}$ is the most active photocatalyst contrastingly with the key control tests.
The trial without catalyst showed negligible annihilation activity, suggesting that the light energy effect (photolysis) of halogen irradiation exerted an unimportant effect on the proliferation of $P$. syringae. Dark tests of the silver-titanates and silver nanoparticles in solution were developed to check the biocidal capability of the AgNPs in the absence of visible light, attaining a biocidal capability of $20 \%$ for $P$. syringae cells within an hour.

Under visible-light irradiation conditions for $P$. syringae, the annihilation of silver-nanotube composites was achieved in less than $60 \mathrm{~min}$, but only with the Nt1Ag and Nt3Ag composites with different performances. Nt0.5Ag only achieved $60 \% P$. syringae cell annihilation. Under dark conditions, the three loadings on nanotubes suffered a notable decay of activity, particularly for Nt3Ag above $50 \%$, followed by Nt1Ag, suggesting the significance of synergic light photoactivation on AgNPs functionalized on the surface of the titanate $\mathrm{Nt} 1 \mathrm{Ag}$, which possessed a specific surface area of $260 \mathrm{~m}^{2} \mathrm{~g}^{-1}$. In the case of the composite with low loading ( $\mathrm{Nt} 0.5 \mathrm{Ag}$ ), the decay of activity resulted in only $11 \%$ cell annihilation. Perhaps the $0.5 \mathrm{AgNP}$ loading was not enough for both biocidal and photoactivity 


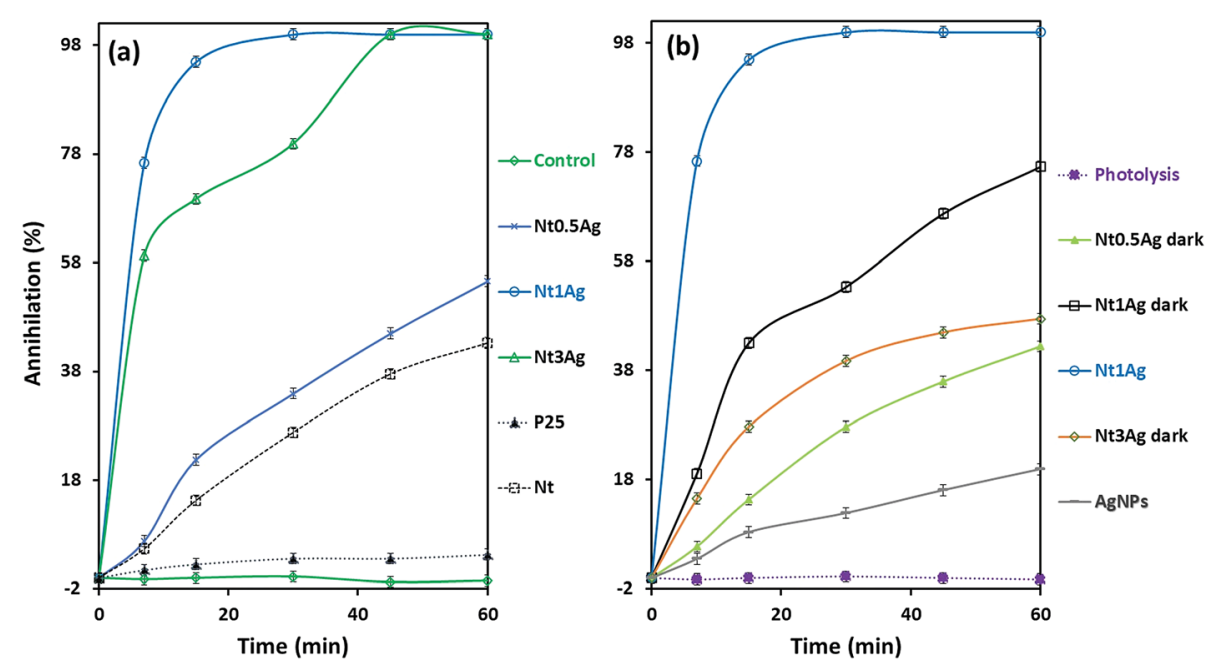

Fig. 4 Photoassisted inactivation of $P$. syringae as a function of time for (a) three loadings of AgNPs on titanate nanotubes, titanate nanotubes alone and commercial $\mathrm{TiO}_{2}-\mathrm{P} 25$ and (b) the most active photocatalyst $\mathrm{Nt1} A g$ in contrast with all the controls.

enhancement effects. Single titanate nanotubes exerted an annihilation effect that was lower than $43 \%$; finally, the commercial $\mathrm{TiO}_{2}$ P25 only achieved $5 \%$ of annihilation of $P$. syringae cells. In previous research with silver titanate nanotubes, the annihilation of Escherichia coli bacteria was achieved within 60 min using a silver loading of $3 \%,{ }^{25}$ and the Botrytis cinerea fungus was annihilated within $20 \mathrm{~min} .^{23}$ Other authors have used $\mathrm{TiO}_{2}$-based nanotubes and achieved the inactivation of $E$. coli and other bacteria in 15 min under a measured light intensity of $3.6 \mathrm{~mW} \mathrm{~cm}^{-2}$ in the UV range $(\lambda=360 \mathrm{~nm})$; UV-light normally exerts an important photolysis effect on bacteria, by the application of potential, 1.5 VSCE anodic bias, which is an electrochemically assisted photocatalytic treatment of bacteria. $^{27} \mathrm{TiO}_{2}$ nanoarrays modified with $\mathrm{Ag}$ displayed a significant capacity for $E$. coli inactivation under UV irradiation, achieving $80 \%$ reduction of the initial $\mathrm{CFU} \mathrm{mL} \mathrm{m}^{-1}$ in 90 min. ${ }^{28}$ As far as we know, our work is the first one to report that $P$. syringae is completely eradicated under solar irradiation by using sustainable hydrothermally synthetized titanate nanotubes functionalized with $4 \mathrm{~nm}$ AgNPs within $30 \mathrm{~min}$. According to previous studies, no specific effect of silver nanotube composites on a specific microorganism seems to occur. HRTEM and SEM were used for assessing the photoassisted inactivation mechanism of $P$. syringae.

\subsection{Morphology and structure of $P$. syringae assessed with TEM and SEM during photocatalytic annihilation}

$P$. syringae is a Gram-negative, strictly aerobic bacterium which is rod-shaped, with polar flagella and dimensions of $0.8-1.5 \mu \mathrm{m}$ and $0.6-6.7 \mu \mathrm{m} .{ }^{9}$ Fig. 5a displays the control P. syringae without treatment and its cells showed dense straight rod shapes with EPS (extracellular polymeric substances). P. syringae was exposed to the Nt1Ag composite and complete photocatalytic annihilation occurred within 20-50 min, which was confirmed by all possible controls, Fig. 4b. P. syringae was analyzed by SEM at different representative time intervals: $5,10,15,30,45$, and
$60 \mathrm{~min}$, in order to reveal the cell damage and possible cell lysis. Fig. 5b-d display the morphological changes undergone by $P$. syringae (Fig. 5b); first, the bacterium shows EPS and some dead cells marked with white arrows. Then, dead cells increased with time, showing a possibly dispersed cell content (Fig. 5c). The white arrows show two kinds of dead cells; one of them shows an oval shape with a big central hole and the second one displays no characteristic oval shape and some of its cellular content is spread outside the cell. In both cases, it is impossible that the cells are viable. In Fig. 5c, black asterisks mark the presence of dispersed cell content; there are deposits of dense material and membranes without shape, which was confirmed by TEM characterization. Finally, only aggregated dead cells were observed (Fig. 5d). The EDS spectrum inset in Fig. 5d displays the semiquantitative composition of Nt1Ag, showing its interaction with the bacterial cells. As a further approach and to visualize in more detail the dead cells interacting with the silver-titanate nanotubes and check their possible penetration in cells, TEM investigation was used.

P. syringae pathogenic bacteria at $0 \mathrm{~min}$ of photocatalytic treatment, as control, are shown in Fig. 6a, in which the cells show an oval shape with some pili and flagellae; the outer membrane (OM), periplasm (P), and plasma membrane (PM) are intact. Fig. $6 \mathrm{c}$, which displays the cell membrane amplified from Fig. 6a, shows cells with a straight rod shape, a capsule, and a well-defined outer membrane. Fig. $6 \mathrm{~b}$ shows several blebs or vesicles that were formed on the outer membrane in $15 \mathrm{~min}$; this action is a typical immediate response mechanism of Gram-negative bacteria that is followed by EPS. ${ }^{2,29}$ Fig. $6 \mathrm{~d}$ shows clearly the production of outer membrane vesicles (OMVs) on the surface of bacteria. ${ }^{30}$ Fig. 6e shows that the interaction with the nanotubes caused cellular lysis and blebbing in some cells within 10 min by disruption of the outer membrane and plasma membrane. Fig. $6 \mathrm{~g}$ displays the disruption of a cell membrane amplified from Fig. 6e. Fig. 6f shows detritus of dead cells without inner content; the cell collapses after one hour, when 

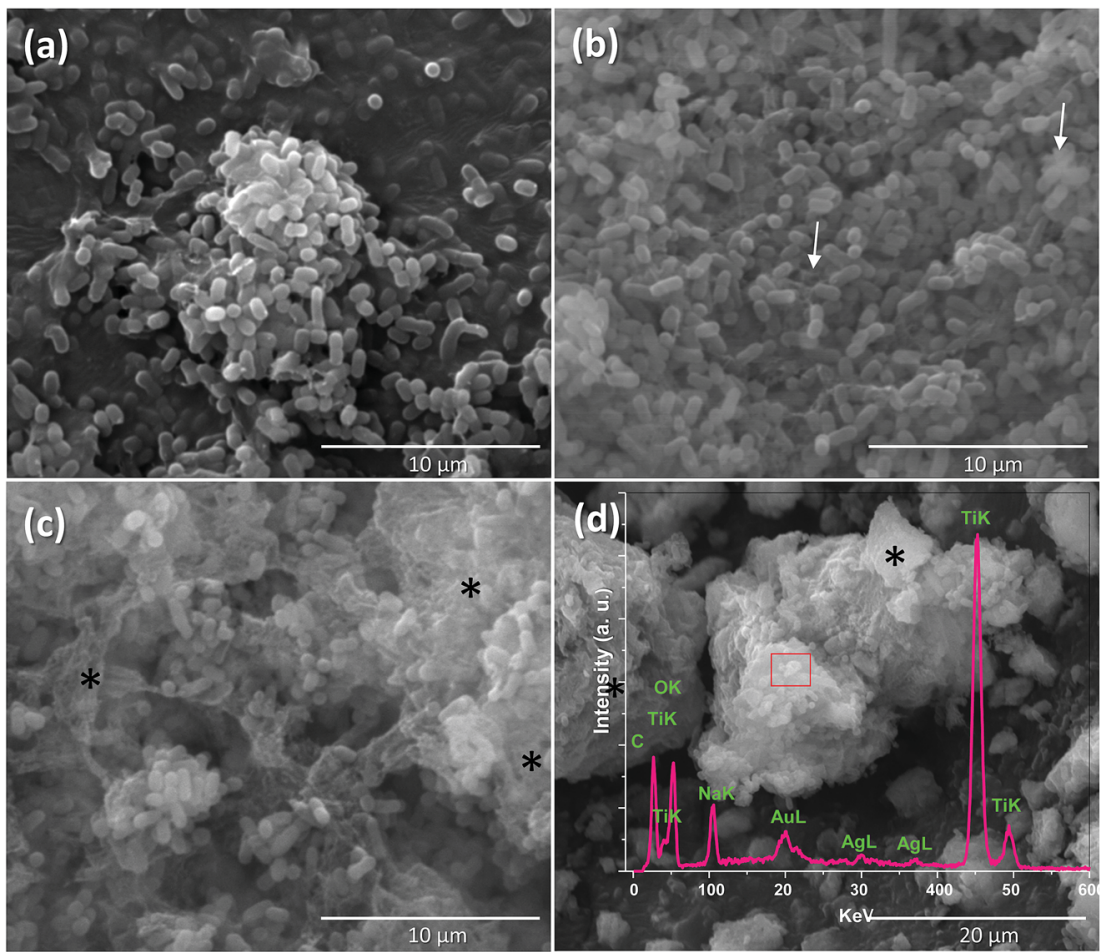

Fig. 5 Effect of silver titanate nanotubes on P. syringae observed by SEM. (a) Control; (b) first stage of EPS damage in 10 min; (c) late stage of damage, emptying of the cell content, $30 \mathrm{~min}$ and (d) massive dead cells within 60 min. Exopolysaccharides (EPS) (white arrows); dispersed cell content (black asterisks).

the annihilation is complete. Fig. $6 \mathrm{~h}$ reveals an amorphous cell membrane that is characteristic of dead cells. A schematic representation is shown in Scheme 1 from the early stage of cell affectation with EPS secretion to the dispersed cell content and destruction of the pathogenic bacteria. In the case of $P$. syringae cells irradiated only with visible-light and interacting with commercial $\mathrm{TiO}_{2}-\mathrm{P} 25$, most of them were only stressed and presented similar disrupted injuries such as those observed in Fig. $6 \mathrm{~b}$ due to the immediate response mechanism of Gramnegative bacteria.

To verify the Ag content and quantify silver lixiviation in the titanate nanotubes, inductively coupled plasma optical emission spectroscopy (ICP-OES) using a 730-ES spectrometer from Varian Inc. was used. $1.27 \mathrm{ppm}$ of silver after $1 \mathrm{~h}$ of sonication, $50 \mathrm{mg}$ of the sample with a higher loading of AgNPs, Nt3Ag, in $50 \mathrm{~mL}$ of deionized water, and $21.8 \mathrm{ppm}$ in the case of Ti were detected. ${ }^{22}$

Photocatalytic decomposition of the $\mathrm{RhB}$ in an aerated environment was performed to get some insights into the role played by the silver nanoparticles in ROS generation and photooxidation, which would help understand their role in P. syringae annihilation.

\subsection{Photocatalytic evaluation of silver-nanotube composites}

In the photodecomposition of Rhodamine $\mathrm{B}(\mathrm{RhB})$ under visible-light, air was bubbled during degradation to try to increase ROS on the surface and promote photo-oxidation reactions. Only the silver composites showed activity for RhB oxidation, and Nt1Ag was the most active one, reaching almost $40 \%$ degradation. All the catalysts followed pseudo-first-order kinetics (Fig. 7a). The photocatalytic RhB performance of the $\mathrm{Nt} x \mathrm{Ag}$ materials was similar to that reported in the literature for nitrogen and iron modified titanate nanotubes. ${ }^{31,32}$ The photocatalytic decomposition of $\mathrm{RhB}$ using $\mathrm{Nt} 1 \mathrm{Ag}$ and $\mathrm{Nt} 0.5 \mathrm{Ag}$ nanotubes after $180 \mathrm{~min}$ of visible irradiation reached percentages of 40 and $30 \%$, respectively, with similar performance (see Fig. 7b). For the P25-1Ag photodeposited commercial $\mathrm{TiO}_{2}$, the percentage was around $36 \%$. This means that the morphology of the photocatalyst is not crucial for dye degradation. The possible mechanism for this reaction is the oxidation of the surface hydroxyl groups or water by the valence band holes and the defects in the crystalline phase structure. Bandgap energy values are not fundamental to explain the enhanced behavior, because for P25, the band-gap energy was $3.2 \mathrm{eV}$ and for $\mathrm{Nt} 1 \mathrm{Ag}$, it was $3.1 \mathrm{eV}$.

The visible-light photodegradation of $\mathrm{RhB}$ revealed that the AgNPs on the surface of nanotubes act as a Schottky barrier, suppressing the rates of photocatalytic recombination, where $\mathrm{TiO}_{2}-\mathrm{P} 25$ and titanate nanotubes were practically inactive for the degradation of RhB under $450 \mathrm{~nm}$ irradiation. When silver was incorporated with identical loadings, $\mathrm{Nt} 1 \mathrm{Ag}$ and $\mathrm{TiO}_{2}-\mathrm{P} 25-$ $1 \mathrm{Ag}$ achieved the maximum degradation. Although $\mathrm{TiO}_{2}-\mathrm{P} 25$ was almost unreactive in the inactivation of $P$. syringae, the role of the sharp tubular morphology of silver-titanate composites in pathogenic bacterium annihilation is important, considering the high surface area that was confirmed through the 

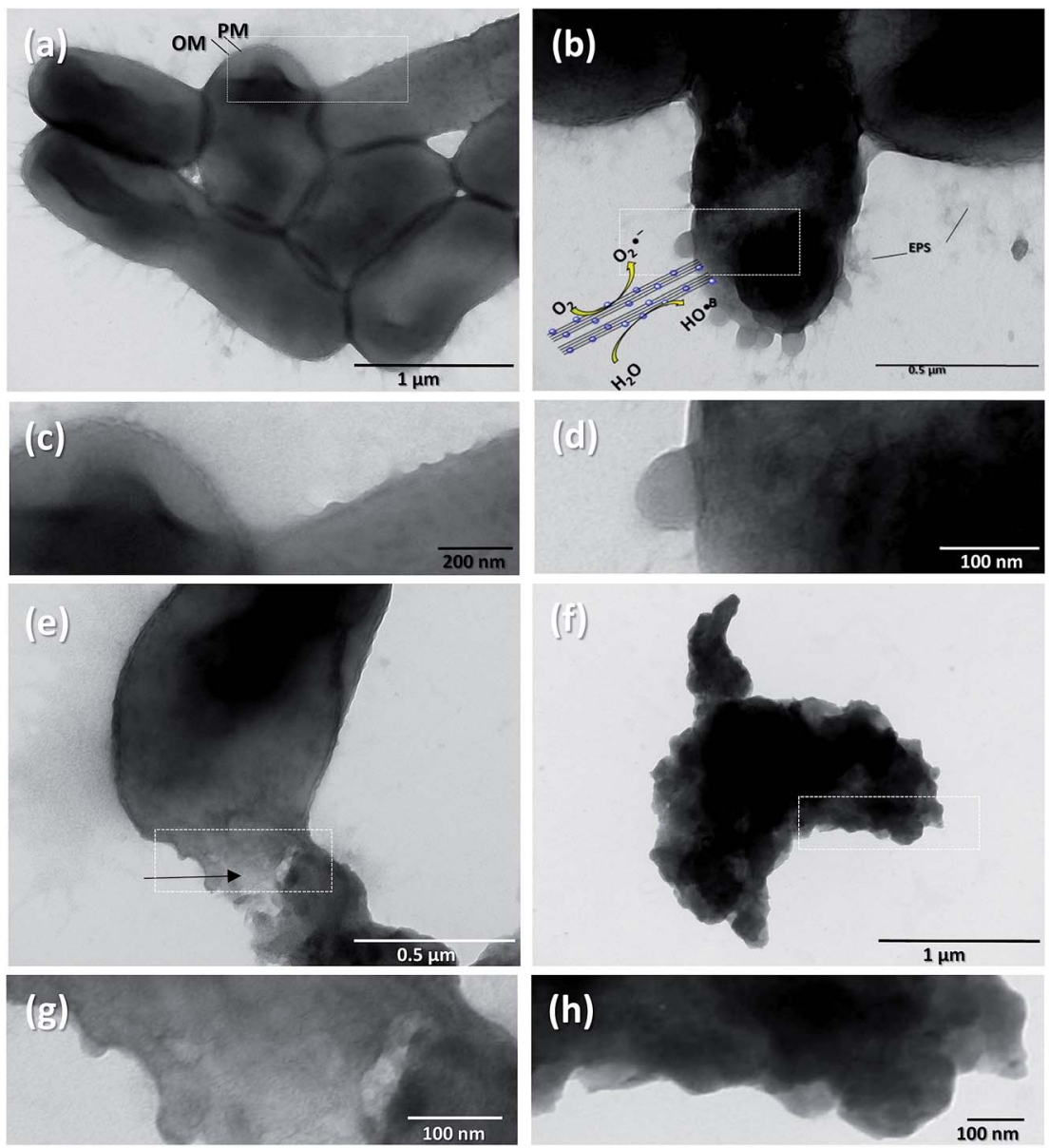

Fig. 6 Effect of the silver nanotube composite Nt1Ag on P. syringae studied by negative staining: (a) control; (b) EPS and OMV formation within 15 min; (c) and (d) enlarged from (a) and (b), respectively; (e) OM and PM disruption (black arrow); (f) detritus of dead cells after one hour; (g) and (h) enlarged from (e) and (f), respectively. $\mathrm{OM}=$ outer membrane; $\mathrm{PM}=$ plasma membrane; $\mathrm{B}=\mathrm{OMVs}$; $E P S=$ exopolysaccharides.

accelerated annihilation, 294 versus $56 \mathrm{~m}^{2} \mathrm{~g}^{-1}$, respectively. ${ }^{23,26}$ High monodispersion of AgNPs on the titanate surface is necessary to optimize the surface oxidation reactions and Schottky barrier function.

The photo-annihilation results correlated with the bacterium morphological injury during annihilation suggest that the possible mechanism that renders $P$. syringae inactive, shown in Scheme 1, may follow three methods: (i) the photoactive generation of ROS when irradiated with visible-light that may stress the cell body and damage the organic composition by oxidation and reduction of the secretions and the external wall, avoiding damage to the host plant; ${ }^{23}$ (ii) the surface functionalization of titanate nanotubes with silver nanoparticles not only boosted the generation of ROS and redox reactivity, but also helped achieve an annihilation of $56 \%$, considering the titanate nanotube activity as the control. This nanocomposite under dark conditions achieved $75 \%$ annihilation, which is indicative of a synergistic effect of photoactivity and biocidal effects; (iii) physical damage to the $P$. syringae cell capsule. SEM and TEM observations clearly showed the physical deformation of the cell wall; however, nanotube penetration was clearly not demonstrated. The main physical damage probably was the laceration of the cell wall caused by the sharp morphology of the nanotubes that may have facilitated cell death, Scheme $1 e^{23,25}$

The bacterium produces compounds as virulence factors that wound the plant; ; ${ }^{2,9}$ likewise, as part of its defense mechanism, it secretes external proteins which were identified by SEM and TEM. The production of compounds in response to environmental stress and as a first resistance mechanism gives rise to possible cell disruption of the bacterium defense mechanism that causes the production of outer membrane vesicles on bacteria. The OMVs and deformation of the outer membrane by disruption were expected to prevent the penetration of nanoparticles; however, the morphology of nanotubes permeated the outer membrane, thus accelerating the annihilation of bacteria and stopping the proliferation irreversibly. This is an important advantage in contrast with fungicides and antimicrobial agents. Chemical compounds need to enter cells via the cell wall and membrane to exert their activity, and some of the compounds are already diluted in biofilm formation. The constant ROS generation by visible irradiation of composites, the bacteriostatic effect of surface AgNPs and the sharp nanotube morphology that produces mechanical stress are key to preventing the bacterial resistance of $P$. syringae. 


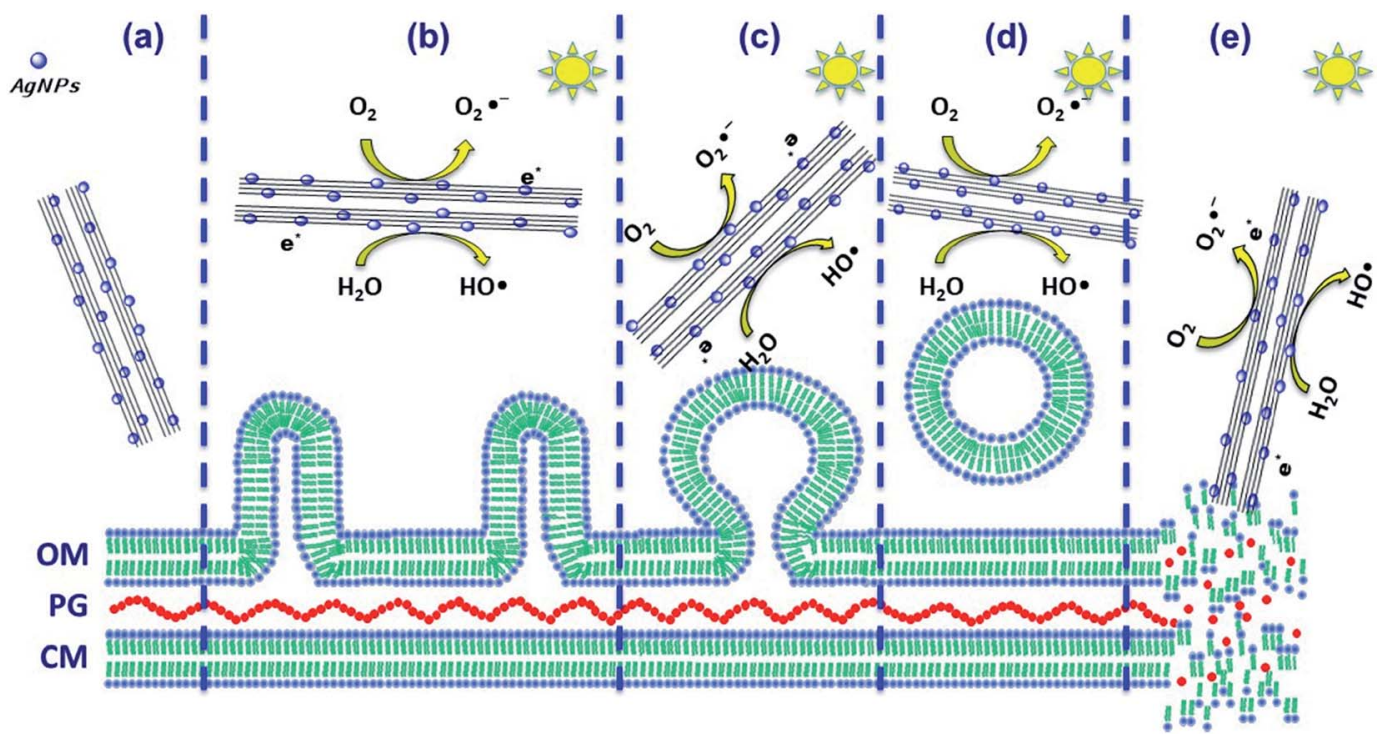

Scheme 1 Pseudomonas syringae: different stages of cell damage during photoinactivation. (a) Before photocatalytic treatment, (b) beginning of ROS generation under visible-light irradiation and 1st stage of bleb formation, (c) 2nd stage of OMVs formation and secretion and bigger and quasi-spherical vesicles, (d) 3rd stage of OMV separation from membranes and (e) complete disruption ending with cell lysis. Schematic representation of the boosted generation of ROS and redox reactivity.

\subsection{Photocatalysis as a tool for pathogenic microorganism eradication}

The action of silver nanotube composites is not microorganism specific; it depends on the loading of silver nanoparticles and biological characteristics of the pathogenic microorganism, and most of the pathogenic microorganisms were annihilated in $1 \mathrm{~h}$. The importance of examining the morphological changes of microorganism cells by SEM and TEM by means of a reproducible biological protocol for sample preparation provides key insights to understand the annihilation mechanism; in the case of $P$. syringae, the formation of OMVs is a resistance mechanism of the cell against cell lysis, which leads to the annihilation of the bacteria within $30 \mathrm{~min}$. The well-known E. coli displays membrane damage, inhibition of EPS and formation of many aggregates of dead cells; the titanate morphology that damaged the integrity of the capsule promotes the elongation of the cell size and density loss in the central part of some cells. The phytopathogenic fungus B. cinerea was annihilated by manifesting plasmalemma invagination that expanded the conidia to induce cell death. ${ }^{23}$ The penetration by mechanical stress was not evident from the above studies; nevertheless, the induction of cell death was achieved in a short time with photochemical, mechanical and biocidal properties at the same time, which meant that the development of the microorganism resistance seemed impossible. Further experiments using molecular and biochemical
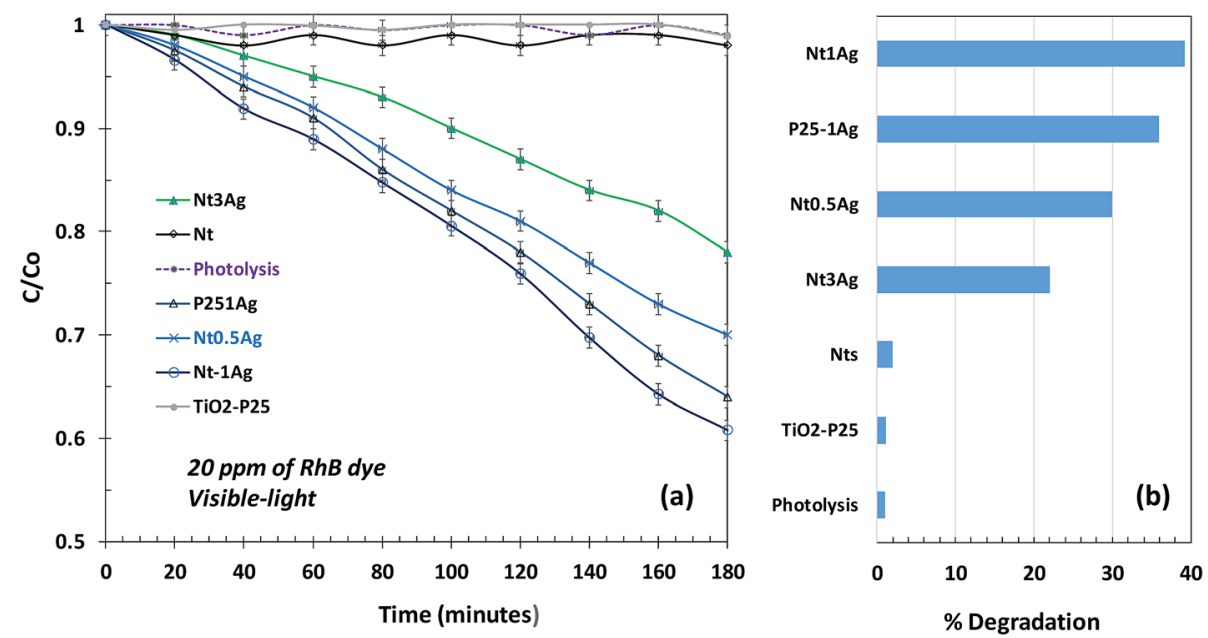

Fig. 7 Photocatalytic degradation of RhB under visible-light irradiation for revealing the surface oxidation reactions: (a) fitted line for $C / C_{0}$ and (b) a bar graph showing \% degradation. 
tools are still needed in order to unravel the intracellular alteration and the signal transduction pathways that are involved during the $P$. syringae response to AgNPs. An effective control strategy in a very short time acts in three simultaneous ways: through photochemical reactions, mechanical damage by the nanotubular morphology and silver biocidal properties made pathogenic cell microbial resistance impossible with time, inhibiting its propagation.

\section{Conclusions}

New reactivity insights into photocatalytic phenomena at the microorganism-nanomaterial interface were possible by means of a reproducible biological SEM and TEM protocol for sample preparation that helped understand the annihilation mechanism of silver titanate nanotubes, more specifically by Nt1Ag. The formation of blebs and/or outer membrane vesicles (OMVs) is a resistance mechanism of the $P$. syringae cells against cell lysis, which leads to the annihilation of the bacteria within $30 \mathrm{~min}$. The synergistic mechanism of silver functionalized nanostructures challenged the defense mechanisms of bacteria and induced fast irreversible cell death of pathogens by a tool aimed at preventing the development of bacterial resistance.

\section{Conflicts of interest}

There are no conflicts to declare.

\section{Abbreviations}

P. syringae Pseudomonas syringae

CFU Colony-forming unit

ROS Reactive oxygen species

OMV Outer membrane vesicles

RhB Rhodamine B dye

VSCE Potential applied on a saturated calomel electrode

\section{Acknowledgements}

We gratefully acknowledge M. S. B. Escoto-Rivera and Dr H. Silva-Pereyra from LINAN-IPICYT for the HRTEM and XRD materials characterization. The authors thank N. A. RamirezPérez and Dr N. Gómez-Hernandez for their technical help during the inactivation experiments. This work was supported by CONACyT grants CB-2011/169597, CB-2013/220791 and LINAN-0271911.

\section{References}

1 J. Van Der Wolf and S. H. De Boer, in Principles of PlantMicrobe Interactions, Microbes for Sustainable Agriculture, 2015, pp. 65-77.

2 S. S. Hirano and C. D. Upper, Microbiol. Mol. Biol. Rev., 2000, 64, 624-653.
3 A. Dudnik and R. Dudler, BMC Microbiol., 2014, 14, 304.

4 I. Yucel, Y. X. Xiao and S. W. Hutcheson, Appl. Environ. Microbiol., 1989, 55, 1724-1729.

5 C. T. Bull, C. R. Clarke, R. Cai, B. A. Vinatzer, T. M. Jardini and S. T. Koike, Phytopathology, 2011, 101, 847-858.

6 O. Berge, C. L. Monteil, C. Bartoli, C. Chandeysson, C. Guilbaud, D. C. Sands and C. E. Morris, PLoS One, 2014, 9, e105547.

7 J. M. Young, J. Plant Pathol., 2010, 92(1), S1.5-S1.14.

8 S. Serizawa, T. Ichikawa and H. Suzuki, Jpn. J. Phytopathol., 1994, 60, 237-244.

9 G. M. Preston, Mol. Plant Pathol., 2000, 1, 263-275.

10 L. Navarini and R. S. Balardin, Summa Phytopathol., 2012, 38, 294-299.

11 H. Fones and G. M. Preston, FEMS Microbiol. Rev., 2013, 37, 495-519.

12 J. Riehl Koch, A. Scherzer, S. Eshita and K. Davis, Plant Physiol., 1998, 118, 1243-1252.

13 S. Ghods, I. M. Sims, M. F. Moradali and B. H. A. Rehma, Appl. Environ. Microbiol., 2015, 81, 4026-4036.

14 J. D. Janse and M. Wenneker, Plant Pathol., 2002, 51, 523536.

15 M. W. Aktar, D. Sengupta and A. Chowdhury, Interdiscip. Toxicol., 2009, 2, 1-12.

16 H.-H. Hwang, S. B. Gelvin and E.-M. Lai, Front. Plant Sci., 2015, 6, 265.

17 A. Abdel-Megeed, J. Pure Appl. Microbiol., 2013, 7, 11211129.

18 M. F. Brugnera, M. Miyata, C. Q. Fujimura Leite and M. V. B. Zanoni, J. Photochem. Photobiol., A, 2014, 278, 1-8.

19 A. J. Huh and Y. J. Kwon, J. Controlled Release, 2011, 156, 128-145.

20 H. A. Foster, D. W. Sheel, P. Sheel, P. Evans, S. Varghese, N. Rutschke and H. M. Yates, J. Photochem. Photobiol., A, 2010, 216, 283-289.

21 A. Kubacka, M. Ferrer, A. Martínez-Arias and M. FernándezGarcía, Appl. Catal., B, 2008, 84, 87-93.

22 Q. L. Feng, J. Wu, G. Q. Chen, F. Z. Cui, T. N. Kim and J. O. Kim, J. Biomed. Mater. Res., 2000, 52, 662-668.

23 V. Rodríguez-González, R. B. Domínguez-Espíndola, S. Casas-Flores, O. A. Patrón-Soberano, R. Camposeco-Solis and S.-W. Lee, ACS Appl. Mater. Interfaces, 2016, 46, 3162531637, DOI: 10.1021/acsami.6b10060.

24 Y. Ge, J. Wu, H. Li, K. Zhang and X. Li, J. Nanosci. Nanotechnol., 2016, 16, 8748-8759.

25 A. Patrón-Soberano, B. P. Núñez-Luna, S. Casas-Flores, A. De Las Peñas, R. B. Domínguez-Espíndola and V. RodríguezGonzález, Photochem. Photobiol. Sci., 2017, 16, 854-860, DOI: 10.1039/c6pp00237d.

26 V. Rodríguez-González, S. Obregón-Alfaro, L. M. LozanoSánchez and S.-W. Lee, J. Mol. Catal. A: Chem., 2012, 353354, 163-170.

27 N. Baram, D. Starosvetsky, J. Starosvetsky, M. Epshtein, R. Armon and Y. Ein-Eli, Appl. Catal., B, 2011, 101, 212219. 
28 K. Mao, Y. Li, H. Zhang, W. Zhang and W. Yan, Clean: Soil, Air, Water, 2012, 41, 455-462, DOI: 10.1002/clen.201100698.

29 V. R. F. Matias, A. Al-Amoudi, J. Dubochet and T. J. Beveridge, J. Bacteriol., 2003, 185, 6112-6118.

30 I. R. Obi, R. Nordfelth and M. S. Francis, Biochem. J., 2011, 439, 321-332.
31 P. Chen, Y. Cai, J. Wang, K. Wang, Y. Tao, J. Xue and H. Wang, Powder Technol., 2018, 326, 272-280, DOI: 10.1016/j.powtec.2017.12.010.

32 C. Yan, K.-F. Chen, C.-H. Lai, S.-W. Lai, Q. Chang and Y.-P. Peng, J. Environ. Sci., 2014, 26, 1505-1512, DOI: 10.1016/j.jes.2014.05.017. 